\title{
Transition Response Characteristics of Heat-Assisted Magnetic Recording and their Performance with MTR Codes
}

\author{
Rathnakumar Radhakrishnan*, M. Fatih Erden ${ }^{\dagger}$, Ching $\mathrm{He}^{\dagger}$ and Bane Vasic* \\ ${ }^{*}$ Department of Electrical and Computer Engineering, University of Arizona, Tucson, AZ-85705 \\ ${ }^{\dagger}$ Seagate Technology, Pittsburgh, PA-15222 \\ Email: \{rathna, vasic\}@ece.arizona.edu, \{fatih.erden, ching.he\}@ seagate.com
}

\begin{abstract}
The response of a Heat-Assisted Magnetic Recording (HAMR) system is very sensitive to the laser spot position. The response is determined by transition characteristics like the center, curvature and length. In this paper, by using the thermal Williams-Comstock model and the microtrack model, the effects of laser spot position on transition characteristics are investigated for both longitudinal and perpendicular recording from a read channel systems perspective. The general trend in their variation is determined and used to explain the resultant change in response. By simulation, we determine the post-Viterbi dominant error events for longitudinal HAMR system. Finally, we apply the well-known MTR codes to mitigate such errors and present their bit error performance.
\end{abstract}

\section{INTRODUCTION}

Heat-Assisted Magnetic Recording (HAMR) is a very attractive alternative to increase the areal density beyond the super-paramagnetic limit. In HAMR, the medium is heated by a laser during magnetization and is subsequently cooled rapidly to room temperature. Since, coercivity decreases with temperature, it results in sharper transitions. The transition response of the HAMR system varies widely not only with the temperature profile [1] but also with the laser spot position [2]. Both the curvature and length of a transition affect the readback signal characteristics. In this paper, we establish the general trend in the variation of transition response of a HAMR system with laser spot position. Also, we determine the dominant error events for longitudinal HAMR generalized partial response channels. Their bit error performance with Maximum Transition Run (MTR) code, used to avoid certain dominant error events, is determined. In Section II, the thermal Williams-Comstock model is introduced and in Section III, the effect of change in laser spot position on transition characteristics is investigated. In Section IV, we discuss the performance of the HAMR system with MTR codes and draw some conclusions in Section V.

\section{Thermal Williams-Comstock Model}

In a magnetic system, the relation between the applied field $H_{a}$ and the resultant magnetization $M$ is given by the hysteresis loop of the medium. Williams and Comstock showed that $M$ can be determined using the well-known slope equation. In [2], this model was extended by incorporating thermal gradients of coercivity $\left(H_{c}\right)$ and magnetization for a longitudinal HAMR system and is known as the Thermal Williams-Comstock model (TWCM). Following the same approach, the slope equation is derived as

$$
\frac{d M(x)}{d x}=\frac{d M(x)}{d H_{a}}\left[\frac{d H_{h}(x)}{d x}+\frac{d H_{d}(x)}{d x}-\frac{d H_{c}(T)}{d T} \frac{d T(x)}{d x}\right]
$$

where, $H_{h}$ is the head field, $H_{d}$ is the demagnetizing field, $T(x)$ is the temperature profile in the medium. A magnetic transition is said to occur when $H_{h}+H_{d}=H_{c}$. In order to solve (1), the transition profile is assumed to take the form of an arctangent, which is completely characterized by two quantities; transition parameter $a$ and transition center (or location) $x_{0} . H_{a}$ and $H_{d}$ are evaluated for longitudinal HAMR as in [2] to solve for $M$.

We extend this model to characterize perpendicular HAMR and present only certain important field expressions due to space constraints. The transition profile, as before, is assumed to be arctangent. An approximate analytical head field expression can be derived [3], by simplifying the perpendicular system to a longitudinal system turned sideways. Taking into account the change in coordinate system, the magnetizing component of the head field is given as

$$
H_{y}(x, y)=\frac{H_{0}}{\pi}\left[\tan ^{-1}\left(\frac{y+g / 2}{x}\right)-\tan ^{-1}\left(\frac{y-g / 2}{x}\right)\right]_{(2)}
$$

where $g$ is the gap width between the pole head and its image. Following a similar approach as in [2], $H_{d}$ is calculated as

$$
\begin{gathered}
H_{d}(x)=\frac{-2}{\pi^{2}} \int_{-\infty}^{+\infty}\left[\frac{M_{r}\left(x^{\prime}\right) a}{a^{2}+\left(x_{0}-x^{\prime}\right)^{2}}+\tan ^{-1}\left(\frac{x^{\prime}-x_{0}}{a}\right)\right. \\
\left.\frac{d M_{r}(T)}{d T} \frac{d T\left(x^{\prime}\right)}{d x^{\prime}}\right] \tan ^{-1}\left(\frac{2\left(x-x^{\prime}\right)}{t}\right) d x^{\prime}
\end{gathered}
$$

The HAMR channel is simulated using the method of microtrack modeling. In this model, a magnetic track is divided into several sub-tracks of equal width. For each sub-track, the thermal Williams-Comstock model is applied independently to determine $a$ and $x_{0}$. The transition responses of each sub-track for longitudinal and perpendicular recording are expressed as Lorentzian and error functions respectively. Though the TWCM model ignores certain thermal effects, it is sufficient to determine important characteristics of HAMR from a read channel perspective [1]. 
TABLE I

SYSTEM PARAMETERS

\begin{tabular}{ll} 
Coercivity $\left(H_{c}(x)\right)$ & $-2000 \cdot T(x)+16 \cdot 10^{5} \mathrm{~A} / \mathrm{m}$ \\
Remnant magnetization $\left(M_{r}(x)\right)$ & $-1200 \cdot T(x)+12 \cdot 10^{5} \mathrm{~A} / \mathrm{m}$ \\
Coercive squareness $(\mathrm{S})$ & 0.7 \\
Deep gap field $\left(H_{0}\right)$ & $19 \cdot 10^{5} \mathrm{~A} / \mathrm{m}$ \\
Write head gap & $100 \mathrm{~nm}$ \\
Distance from pole to medium & $20 \mathrm{~nm}$ \\
Read head gap & $5 \mathrm{~nm}$ \\
Width of the track & $120 \mathrm{~nm}$ \\
Number of microtracks & 17 \\
\hline
\end{tabular}

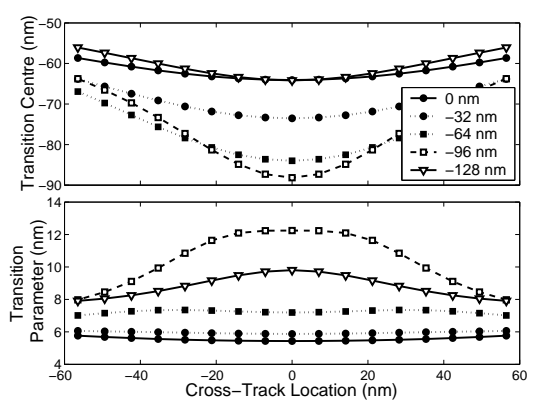

Fig. 1. Transition centres and parameters across the track for various laser spot positions to the left of the gap center $(0 \mathrm{~nm})$ for Longitudinal HAMR

\section{EFFECTS OF LASER SPOT POSITION}

Along the track, the laser can be positioned either in the direction of the head movement (up-track) or opposite to it (down-track). For all cases, the laser is assumed to be at the center of the track in the cross-track direction. The behavior of the system is shown with the help of a specific example but the reasoning itself, as will be seen, adheres to a wide range of HAMR systems. We first discuss the Longitudinal HAMR system. The temperature induced by the laser is assumed to be Gaussian in both dimensions with peak $400{ }^{\circ} \mathrm{C}$ and width $70 \mathrm{~nm}$. Other parameters are as given in Table I. Fig. 1 shows the transition centers across the track at different laser spot positions in the down-track direction. It reveals two general trends. As the laser is moved down-track from the gap center $(0 \mathrm{~nm})$, transition centers initially move away from the gap center until they occur at the positive coercivity gradient region. Thereafter, moving the laser further downtrack, moves the centers back closer to the gap center. As the transition location moves away from the gap center, the curvature deteriorates, since the center now occurs closer to the peak temperature, where the variation in thermal gradient across the track is the highest. Consequently, in this example, the transition curvature is at its worst when the laser is aligned at $-96 \mathrm{~nm}$ (location closest to peak temperature) and improves on either side of this position. Fig. 1 also shows the cross-track transition parameter profile. As before, two trends are identified. The parameter generally increases when laser is moved down-track toward the lower gradient region. In the example, at an alignment of $-96 \mathrm{~nm}$ there is a huge increase largely contributed by almost-zero $H_{c}$ gradient and low $H_{h}$. Also, unlike the usual case, the transition parameter is maximum at the center and minimum at the edges. Since,

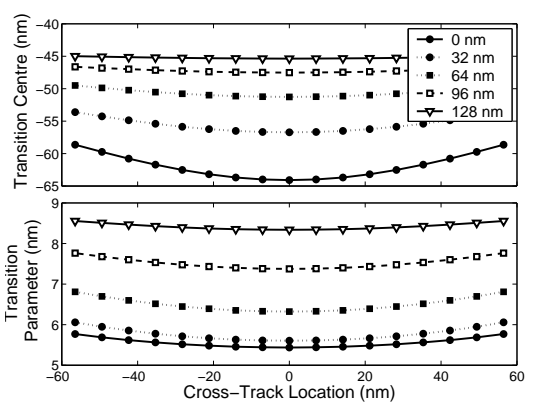

Fig. 2. Transition centres and parameters across the track at various laser spot positions to the right of gap center $(0 \mathrm{~nm})$ in Longitudinal HAMR

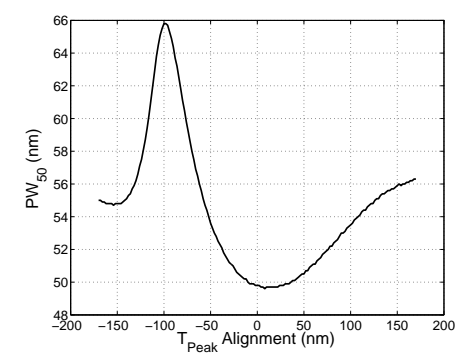

Fig. 3. $P W_{50}$ for various laser spot positions in Longitudinal HAMR

at this laser position the coercivity gradient is positive at the transition location, a higher gradient would result in a higher transition parameter. Toward the edges of the track, the $H_{h}$ gradient increases and $H_{c}$ gradient decreases thereby reducing the transition parameter. At an alignment of $-128 \mathrm{~nm}$, though the profile remains the same, the transition parameter values have improved on account of better $H_{h}$ gradient.

When laser is moved up-track, the transition center always occur where the $H_{c}$ gradient is negative. The farther the laser is from the gap center, the farther the transition center is from the peak temperature. Therefore, as shown in Fig. 2, there is almost no curvature for positions far to the right of the gap center. For the same reason, there is not much change in the transition parameter profile. However, since the transition location is pushed to the lower temperature regions, the transition parameter in general increases. Since, $P W_{50}$ increases with decrease in transition parameter and increase in curvature, the resultant effect of the change in transition characteristics on $P W_{50}$ is as shown in Fig. 3.

Using Eqn. 2 and Eqn. 3, the transition characteristics were studied for perpendicular recording where, $H_{c}(x)$ is $-2000 \cdot T(x)+21 \cdot 10^{5} \mathrm{~A} / \mathrm{m}$ and pole-to-keeper width is $80 \mathrm{~nm}$. Rest of the parameters were same as before. Fig. 4 and Fig. 5 show that the general transition characteristics are quite similar to the longitudinal case. However, note that the transition parameter decreases as the laser is moved uptrack. We believe this is an artifact of the assumed head field expression, as it is not a good approximation around the pole edges. When the transition occurs close to the pole edges, the head field expression results in a very high field gradient, thereby decreasing the transition parameter. 


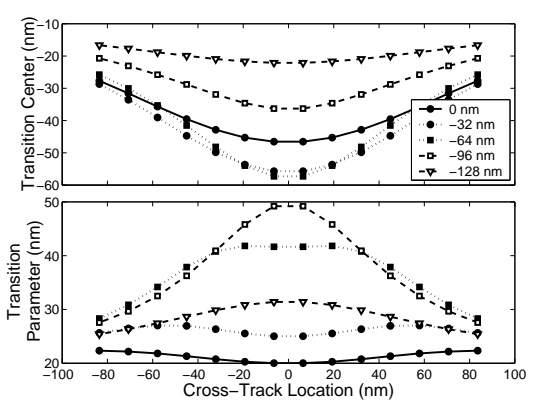

Fig. 4. Transition centers and parameters across the track at various laser spot positions to the left of gap center $(0 \mathrm{~nm})$ in Perpendicular HAMR

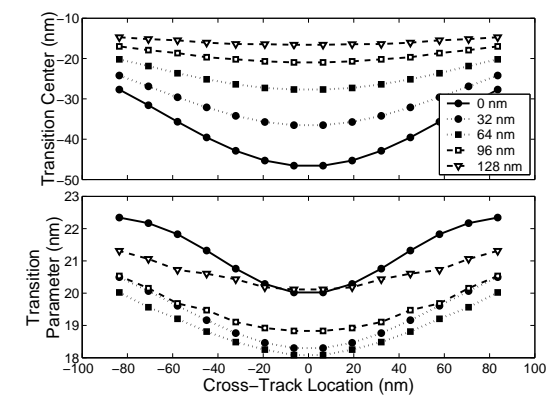

Fig. 5. Transition centres and parameters across the track at various laser spot positions to the right of gap center $(0 \mathrm{~nm})$ in Perpendicular HAMR

\section{Performance with MTR Codes}

A HAMR simulator is developed comprising the numerical HAMR channel implemented using the model described above, followed by a low-pass filter and symbol rate sampler. The channel response is equalized to a generalized partial response target [4] and decoded using the Viterbi detector. The isolated transition response of the HAMR longitudinal system is very similar to the Lorentzian function in most cases of interest. By simulation, it is determined that the dominant error events for the system with electronic noise (AWGN) are $\pm[+1-1+1]$. These error events are induced by three or more consecutive transitions in the input data sequence and has been extensively studied [7]. Traditionally, constraint codes are used to encode the data sequence so as to avoid occurrences of certain error events. MTR codes [5], [6] are a popular choice in literature to avoid tribit error events.

Unlike communication systems, loss due to coding in magnetic recording is roughly proportional to the square of the code rate $\left(R^{2}\right)$; increase in bandwidth and loss due to decrease in dipulse response energy. Often, both are not taken into account while evaluating coding strategies. Bit error rate performance of MTR codes of rate 6/7 and 8/9 in HAMR system with AWGN noise is determined by simulation and is shown in Fig. 6 along with the uncoded performance. When appropriate coding rate losses are taken into account, it is surprising to note that the code rate penalties negate the gain achieved by the code in eliminating tribit error events. Though not shown here, the MTR codes will achieve a gain only at very high SNR's where all errors are due to the tribit error events. Since these codes are almost capacity achieving, it suggests that soft-

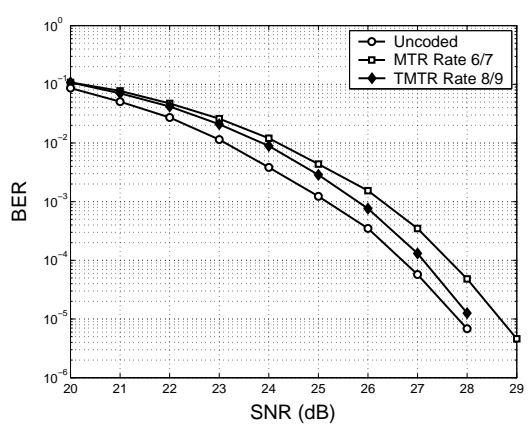

Fig. 6. Bit error rate performance of MTR constraint codes in longitudinal HAMR (normalized density 3 )

constraint codes or post-Viterbi processors should be used to deal with dominant error events in longitudinal HAMR. However, it should be noted that these constraint codes may be more beneficial in media-noise dominated systems, as avoiding consecutive transitions may reduce media noise.

\section{CONCLUSION}

In this paper we extended the thermal Williams-Comstock model (TWCM) to perpendicular HAMR. Using TWCM, effects of laser spot position on the system response were determined. Unsymmetrical change in the width of the response is explained by analyzing the relationship between the temperature profile and transition characteristics like the curvature and length. Consequently, a general trend in the variation of such characteristics is established. Further, tribit errors were found to be the dominant source of error in longitudinal HAMR in the presence of electronic noise. But, poor performance of MTR codes suggests the use of softconstraint codes or post-Viterbi processors to mitigate such errors.

\section{ACKNOWLEDGMENT}

This work was performed as part of the INSIC HAMR ATP program with the support of the U.S. Department of Commerce, National Institute of Standards and Technology, Advanced Technology Program, Cooperative agreement number 70NANB1H3051.

\section{REFERENCES}

[1] M. Fatih Erden, Tim Rausch and William Challener, "Cross-track location and transition parameter effects in Heat-Assisted Magnetic Recording", IEEE Trans Mag, May 2005.

[2] Tim Rausch, James A. Bain, Daniel D. Stancil and Tuviah E. Schlesinger, "Thermal Williams-Comstock model for predicting transition length in a Heat-Assisted Magnetic Recording system”, IEEE Trans Mag, Vol 40, No 1, Jan 2004.

[3] John C. Mallinson and H. Neal Bertram, "On the characteristics of polekeeper head fields", IEEE Trans Mag, Vol 20, No 5, Sept 1984.

[4] Jaekyun Moon and Weining Zeng, "Equalization for maximum likelihood detectors", IEEE Trans Mag, Vol 31, No 2, March 1995.

[5] Barrett Brickner, Jaekyun Moon, "Design of a rate 6/7 maximum transition run code", IEEE Trans Mag, Vol 33, No 5, Sept 1997.

[6] William Bliss, "An 8/9 time-varying trellis code for high density magnetic recording", IEEE Trans Mag, Vol 33, No 5, Sept 1997.

[7] B. Vasic and E. M. Kurtas, Coding and Signal Processing for Magnetic Recording Systems, chapter 18, pp 18.1-18.12, CRC Press, 2004. 